\title{
LA HIPERTEXTUALIDAD DESDE LA INTELIGENCIA SINTIENTE. APROXIMACIÓN DOCUMENTAL
}

\author{
Olga Lucía Londoño Palacio, Ph.D. ${ }^{64}$
}

Yo me atrevo a insinuar esta solución del antiguo problema: La biblioteca es ilimitada y periódica. Si un eterno viajero la atravesara en cualquier dirección, comprobaría al cabo de los siglos que los mismos volúmenes se repiten en el mismo desorden (que, repetido, sería un orden: el Orden). Mi soledad se alegra con esa elegante esperanza.

"La Biblioteca de Babel" Jorge Luís Borges

\section{RESUMEN}

El propósito de este artículo es identificar los trabajos más relevantes relacionados con la forma de llevar a cabo una recopilación histórica desde un sistema de hiperlibro. Para ello, se tomaron como descriptores temáticos, el concepto de meta-sistema, inteligencia artificial y el sistema de marcos, inteligencia sintiente, identidad cultural, Teoría de la Acción Comunicativa y el concepto de narrativas y tipos de narración que darán inicio al proyecto perteneciente a la línea de investigación Inteligencia Sintiente e Identidad Cultural perteneciente al Doctorado en Gestión de Conocimiento.

Palabras clave: metasistema, Desarrollo Regional, Identidad Cultural, Visibilidad Institucional, Inteligencia Sintiente, Ontología Unadista.

\begin{abstract}
The purpose of this article is to identify the most relevant works related to the methodology of historical compilation based on a hypertext system. To this end, the following concepts were used as thematic descriptors: meta-system, artificial intelligence, frame systems, emotional intelligence, cultural identity, Theory of Communicative Action, narratives and narrative types, all of which will give rise to the project belonging to the Emotional Intelligence and Cultural Identity research line, within the Doctorate Program in Knowledge Management.
\end{abstract}

64 Licenciada en Filosofía y Humanismo (Universidad Santo Tomás de Bogotá). Especialista en Investigación Lingüística y Literaria (OFINES, Madrid). Especialista en Filología Española (Universidad de Málaga - España). Magíster en Hábitat (Universidad Nacional de Colombia). Doctora en Filosofía, con énfasis en Antropología (Atlantic International University, La Florida - USA). Actualmente forma parte del equipo que está estructurando el Doctorado en Gestión del Conocimiento en la Universidad Nacional Abierta y a Distancia. 
Key words: meta-system, Regional Development, Cultural Identity, Institutional Visibility, Ambient Intelligence, Unadist Ontology.

Recibido: 26 de agosto de 2009

Aceptado: 23 de octubre de 2009

\section{INTRODUCCIÓN}

Este artículo es una invitación a conocer y a experimentar una nueva forma de narrar desde la memoria de la misma realidad vivida con una inteligencia sintiente, es decir, a partir de la experiencia compartida desde la realidad misma. Dicha narración puede tener diversas formas; quizá, la más frecuente es la verbal pero también se puede narrar mediante imágenes, sonidos, colores u otros sistemas semiológicos, con la idea de hacer representaciones de la historia, la cultura, los contextos, las formas de gestionar conocimiento, entre otras dimensiones, lo que posibilita la formación de los sistemas conducentes a afianzar las identidades regionales.

Si bien el campo temático en torno a la recuperación de memoria pareciera ser bastante extenso también es cierto que sus bases son investigaciones desarrolladas desde diversas perspectivas socioculturales con la intención de aproximarse a la explicación y/o a la comprensión de fenómenos específicos en el ámbito exclusivo de la historia. La contribución en este trabajo se dirige a la creación de un modelo de recuperación de memoria y la forma de representarla, desde un contexto más amplio, pues no sólo se dirige al aspecto geográfico sino que también tiene un componente estructural al estar planteado desde diversas narrativas organizadas de manera hipertextual que permiten una amplitud interdisciplinar, una presentación en red y el modelo encuentra su articulación desde las particularidades que caracterizan la Inteligencia Sintiente.

Esta propuesta contribuye a identificar el tipo de estudios, preguntas y avances de investigación en torno a la revisión documental que en un inicio guiará la Línea Doctoral Inteligencia Sintiente e Identidad Cultural, perteneciente al Doctorado en Gestión de Conocimiento que se está desarrollando en la Universidad Nacional Abierta y a Distancia UNAD; esto es, encontrar entre la pluralidad de lenguajes existentes, aquel que se conciba como óptimo para describir, desde representaciones digitales, los procesos históricos, las diversidades culturales, los modos de gestionar conocimiento, el conocimiento de los espacios y lugares, las formas administrativas para la gestión, los múltiples medios y contextos; en pocas palabras, el ser y la esencia de los actores que conforman las regiones, las instituciones, los espacios o los lugares, reflejados en su mismo hacer, a partir de las manifestaciones que le otorgan identidad.

Es importante subrayar que es el concepto de sistema, visto desde la Inteligencia Sintiente, el que conduce a la necesidad de un mecanismo de representación, que haga visible las características ontológicas que le dan identidad al objeto/sujeto que se decida a interiorizar como motivo de estudio en forma de proyecto a partir de narrativas en las que sea posible apreciar una relación hipertextual cuya función cultural tenga la capacidad de subsumir su propia textualidad con otras textualidades. Con lo anterior se logra una conexión de tipo intertextual, donde el resultado se convierta en generatriz formal y estructural de dicha hipertextualidad. 


\section{Planteamiento del problema}

Para la elaboración de este primer paso de revisión documental, se hace necesario reconocer los lugares temáticos desde donde surgen aquellos términos que servirán a quienes opten por trabajar desde este modelo. Además, se hace necesario indagar sobre la producción académica existente que sirva como una primera aproximación con miras a una futura sistematización, para el grupo de estudiantes de la mencionada Línea de Investigación del Doctorado en Gestión de Conocimiento, obteniendo con ello reconocimiento y visibilidad de dicha producción, pues como bien lo afirman Maldonado y otros (2007), la visibilidad es un factor que incide positivamente en el reconocimiento y en el desarrollo científico de una comunidad.

En los anteriores términos, la pregunta rectora que orienta y posibilita el desarrollo de esta primera aproximación documental es:

- ¿Qué autores, epistemologías, metodologías y temáticas sirven como apoyo para proponer un modelo que represente las identidades de diversos actores a través de narrativas y que a su vez sirvan para el desarrollo de la Línea de investigación del Doctorado, Inteligencia Sintiente e Identidad Cultural?

\section{OBJETIVOS}

\section{Objetivo general}

Reconocer los lugares epistemológicos, teóricos, metodológicos y temáticos, desde los cuales se están produciendo diseños e implementaciones con formato de Hiperlibro, elaborado desde la construcción de narrativas y a partir de un sistema de marcos, el cual autoriza representarlos a través de diversas manifestaciones lingüísticas que dan lugar a su expresión a través de recursos y formatos digitales accesibles en red.

\section{Objetivos específicos}

1. Presentar de manera sintética una lectura analítica de la documentación seleccionada, clasificada y reseñada.

2. Presentar un recuento de la metodología del rastreo documental, precisando los pasos seguidos, las estrategias trazadas para concentrar la información, los criterios que se tuvieron para seleccionarla, la manera como se organizó y la forma como se analizó.

3. Generar nuevas comprensiones y construcciones teóricas que orienten investigaciones futuras y contribuyan a la comprensión global de este tipo de estudios.

4. Ubicar los textos documentales más representativos relacionados con los conceptos básicos que se trabajan en la Línea Doctoral Inteligencia Sintiente e Identidad Cultural.

5. Concretar el manejo conceptual de los términos básicos que serán utilizados durante el desarrollo de la Línea del Doctorado en Gestión de Conocimiento. 


\section{JUSTIFICACIÓN}

Esta revisión documental inicial pretende formar la conciencia en cada actor de que es parte de un gran sistema y que lo que hace se apoya y tiene consecuencias en la historia del contexto en el que se encuentra. Por lo anterior, se aborda este primer momento, enmarcado como una investigación fundamentada en el conocimiento acumulado, el cual tiene un desarrollo propio y cuya finalidad esencial es dar cuenta de construcciones de sentido sobre bases de datos que apoyan un diagnóstico y un pronóstico en relación con el material documental sometido a análisis (Hoyos, 2000).

Así mismo, debido a que el proyecto apunta a potenciar el avance de la Línea de Investigación Doctoral y entendiendo que los logros que se van obteniendo generan experiencia y conocimiento, en este artículo se considera que es importante:

1. Identificar un modo de pensar, a partir del intercambio conceptual donde se dé la posibilidad de analizar, debatir, criticar y elaborar opiniones autónomas.

2. Generar una dinámica de conocimiento para una de las líneas de investigación del Doctorado en Gestión de Conocimiento, Inteligencia Sintiente e Identidad Cultural, desde una visión crítica, que permita la apropiación del saber colectivo que han desarrollado diversos teóricos.

3. Involucrar diversas comunidades para que se interesen, desde el ámbito cultural, en los procesos de producción de conocimiento.

Además, aquí es importante resaltar que el Ministerio de Cultura a través de la Ley 397 de 1997 , define la cultura como el conjunto de rasgos distintivos, espirituales, materiales, intelectuales y emocionales que caracterizan a los grupos humanos y que comprende, más allá de las artes y las letras, modos de vida, derechos humanos, sistemas de valores, tradiciones y creencias. Admite la misma entidad que la cultura tiene diversas manifestaciones identitarias, y que son éstas las que fundamentan la nacionalidad y algunas de las distintas actividades propias de la sociedad colombiana en su conjunto, al ser consideradas como procesos generados de manera individual y colectiva por los colombianos.

\section{CONTEXTUALIZACIÓN TEÓRICA}

\section{El concepto de meta-sistema}

Llevar a la realidad el concepto de meta-sistema implica asumir una estrategia de representación válida para el sistema representado. En la época previa al surgimiento de la informática Vannevar Bush (1945) presenta un sistema ideal, al que denomina Memex, dispositivo más conocido por su influencia en la visión del hipertexto como estrategia para representar un sistema de conocimiento teóricamente ilimitado y a la vez estructurado. El Memex era un dispositivo mecánico en el que un individuo almacenaba archivos tales como libros, grabaciones o comunicaciones. Su mecánica permitía consultar la información acopiada de una forma rápida, flexible y no lineal. En su aspecto físico, tenía la apariencia de una mesa con dos monitores que 
se accionaban a través de un lápiz óptico y un escáner. De esta forma era posible colectar la información textual o gráfica, siguiendo estándares universales.

Quien acuñó los términos hipertexto e hipermedia fue Theodore Nelson (1967). Su propuesta se dirigió a plantear una forma de manejo de la información, no necesariamente lineal, en la que un objeto o texto se relacionaba con otro que se encontraba en un documento diferente, concepto adoptado más adelante en la World Wide Web para facilitar la búsqueda de información. Según Nelson, el hipertexto logra una búsqueda intuitiva y de acuerdo con las necesidades del usuario, disminuyendo con ello el desgaste que ocasiona la pérdida de tiempo de búsqueda de una información. Xanadú fue el nombre de este proyecto, cuyo objetivo consistió en crear un documento que abarcara varios tipos de información para tener acceso a ellos desde un sólo documento a través de su vinculación con una palabra relacionada con el tema.

Una concepción teórica similar a la anterior fue planteada por Paul Baran y J.C.R. Licklider y es la que motiva el diseño de la red telemática que conecta a todo el mundo sin fronteras, la cual es diseñada por Robert Kahn y Vinton Cerf (1972) y reconocida como protocolo informático llamado TCP/IP (Transfer Control Protocol/Internet Protocol). Tenía como objetivo la creación de una red de redes; es decir, una interconexión entre diversas redes, sin importar que fueran de diferente tipo y funcionaran desde sistemas operativos o maneras de conexión distintos.

El lingüista Roland Barthes (1980), cuando pretende responder a la pregunta sobre cuál es el ideal de la textualidad, lo que logra es definir el significado del hipertexto informático, al afirmar que dicho ideal es construir bloques de palabras que al enlazarse ocasionen diversos y múltiples recorridos que a su vez conformen cadenas de trayectos; así, el ideal narrativo es la textualidad abierta, inacabada y sin un centro ni una jerarquía precisos. Afirmando la pluralidad de la lengua, Barthes confirma que los significados son independientes de su contenido por lo que generan sentidos múltiples y posibles de ser combinados. Así, para este autor la posibilidad textual además de ser polisémica es polifónica, y es por ello que permite diversos puntos de vista.

Esta misma idea la sostiene Jaques Derrida (1972), para quien la apertura textual y la intertextualidad no dependen tanto del texto escrito como del lector que se acerca a él, en cuanto a que es este último, quien a través de asociaciones y competencias, tiene la posibilidad de abrir un texto a otros contextos, por lo general diferentes a los presentados por el escritor, causando una discurso discontinuo, una especie de montaje que genera un efecto relacionado aunque distinto. Concluye Derrida que la estructura del lenguaje se asemeja a una red sin límites que conduce una función lúdica a la que denomina deconstrucción, que no es otra cosa que un acontecimiento del sujeto cultural que escribe, producto de las nuevas tecnologías de la información, que es al mismo tiempo real y virtual, y se escribe y se inscribe bajo una nueva textualidad o hipertextualidad.

\section{Inteligencia sintiente}

El término Inteligencia Sintiente, es una paráfrasis de la obra del filósofo español Xavier Zubiri, Inteligencia Sentiente (1980). Retomando la idea de Zubiri, de definir la inteligencia 
como la capacidad exclusivamente humana de aprehender y sentir la realidad para entenderla por medio de los sentimientos, los sentidos no cumplen un papel de sintetizadores de esa realidad sino, mejor, son sus analizadores o modos de sentir intelectivo. Para Zubiri la inteligencia es una forma de pensamiento hecha de emociones en la que se interrelacionan lo metafísico con lo cotidiano. Es este hecho el que permite, además de un arraigo con lo real, el despliegue de la riqueza que de suyo tiene el estar instalados frente a la realidad con sus limitaciones, sus problemas y sus posibilidades, para inteligir las cosas desde su dentro, desde sus mismas entrañas.

En su propuesta, Zubiri dice que una nueva idea de realidad conlleva una nueva idea de inteligencia, y a la inversa, una nueva idea de inteligencia conlleva una nueva idea de realidad. Por ello, su teoría supone la aprehensión directa y no mediatizada sobre los hechos y las cosas, consintiendo actualizar la realidad. La actualidad, afirma el filósofo español, es un estar presente y, la inteligencia es el sentido de esa presencia en la realidad. Por lo tanto, inteligencia y sensibilidad son una sola facultad que permite, frente a la realidad, inteligir aquello que se siente así como a sentir lo que es inteligido. Lo que significa que la realidad se siente cuando es trascendida en forma dinámica a través de la aprehensión de su esencia. O mejor, en palabras de Zubiri (1984: 372):
El hombre se realiza en una forma de realidad que las cosas no le imponen, pero no puede hacerlo más que con y por las cosas. De ahi que las cosas no hacen sino abrir, en el poder de realidad que vehiculan, distintas posibilidades de adoptar una forma de realidad u otra. Por tanto, entre ellas tiene que optar el hombre. Optar no es sólo "elegir" lo determinado de una acción, sino que es "ad-optar" una forma de realidad en la acción que se ha elegido.

La intelección conduce a la apropiación de las cosas para extraer de ellas la riqueza de propiedades que sostienen la realidad del hombre y ofrecen las posibilidades de realización personal. Gracias a la intelección es entonces posible separar, distinguir, clasificar, ordenar, definir, analizar y componer la realidad de las cosas aprehendidas haciendo uso de preceptos, conceptos, juicios, teorías, raciocinios, en una actividad que es elaboración o construcción activa de lo aprehendido. Y, es gracias a los sentidos que los seres humanos tienen intelección, la atemperan y se sienten afectados por las cosas de la realidad. Al sentir, la inteligencia fluye en la realidad conectada con ella a través de las cosas, pues desde la realidad se va a las cosas en un acto de apropiación propio del saber y cuyo acervo final es el conocimiento.

En cuanto a los procesos de investigación que incluyen a la Inteligencia Sintiente, Zubiri (2005) manifiesta que todo investigador busca la verdad, pero no la de las afirmaciones hechas por el investigador sino la de la realidad misma. La investigación, afirma, no es una ocupación sino una dedicación que apunta a que sea la realidad la que configure la mente de quien investiga. La suma de realidades o el estudio de algunos fragmentos de ella puede abordarse desde diversos saberes específicos, tales como la física, las matemáticas, la biología, las ciencias sociales, la filosofía, entre muchas otras, pero esa especificidad le da un carácter incompleto pues, por ser la realidad abierta y múltiple, su abordaje debe hacerse desde las mismas características. Por ello, el investigador es un buscador de la verdad que debe vivir intelectiva y sentientemente, profesando siempre la verdad de la realidad. 


\section{Identidad cultural}

Las orientaciones teóricas en torno a la cultura se enfocan hacia las descripciones y explicaciones de los fenómenos culturales, centrando sus trabajos en lo que consideran importante para ser descrito y, muy posiblemente, para explicar algunos aspectos concretos de una cultura. Esto conlleva un flujo de ideas que confluyen bien en el planteamiento o bien en la resolución de problemas, pues describir las identidades culturales de los pueblos, tales como el arte, la religión o la política, entre otras muchas expresiones, es al mismo tiempo una forma de apreciar las manifestaciones de las formas de vida del ser humano, como creador de sus propias ideologías, símbolos, lenguajes e instituciones.

Afirma García Canclini (1995) que la esencia humana no es una ni es la misma para todos; es decir, debido a que cada pueblo tiene una manera de formalizar la realidad, cada pueblo es también poseedor de una cultura. Si no fuera así, no existirían las diferencias culturales, las cuales se manifiestan en el lenguaje, la religión, las costumbres sociales, el vestido, la alimentación, en fin, en formas de ser, actuar, pensar o sentir. Se puede afirmar que lo único que comparten las culturas es el actor; esto es, el hombre quien es el encargado de darle una identidad, entendida ésta como algo históricamente constituido, imaginado, reinventado, en proceso constante de hibridación y transnacionalización, que disminuye su antiguo arraigo cultural.

En la construcción de identidades, son muchos los referentes que pueden ser incorporados; desde la historia de vida de un individuo hasta la de una comunidad, un pueblo o una región. El referente geográfico es el más tangible y objetivable elemento de identidad, según Salazar (1996) pues, dada la variedad geográfica dentro de los países, es lógico que el papel de lo territorial y todo lo relacionado con él esté más marcado en estas identidades (citado en Zúñiga y Asún, 2003: 75). Algo que caracteriza a los individuos y a las comunidades es utilizar sus cualidades para crear formas de inclusión y exclusión, divisiones entre un ellos y un nosotros, entre los que pertenecen y los que no. Así, las fronteras tienen un doble rol en la construcción del sentido de lugar: existen los de dentro y también los de fuera.

Es Castells (1999) quien explica que la identidad cultural se constituye como reacción social frente a la crisis del estado nación, que desde los años noventa se ha visto enfatizada desde aspectos que van más allá del costumbrismo y cotidianeidad. De acuerdo con Zúñiga y Asún (2003) esta identidad puede definirse como aquella parte del autoconcepto de un individuo por estar basada en su pertenencia a un grupo regional, junto con el significado valorativo y emocional (sintiente) $)^{65}$ asociado a dicha pertenencia y alude a un sentimiento de pertenencia y a un sistema cultural de referencia. Como lo indican los mismos autores, se basa en la conjunción entre el medio físico (base ecológica), la continuidad histórica (base temporal) y la continuidad social (base cultural).

El concepto de identidad cultural es dinámico, y se refiere al proceso a través del cual las personas identifican un territorio y se identifican con él, con su cultura, tradiciones, paisaje, historia, etc. La identidad cultural se puede manifestar de diversas formas, como complejo

65 Este agregado se realiza en el presente trabajo. 
de inferioridad o como orgullo, como sentimiento de pertenencia, derecho y proactividad cultural o política. Asimismo, no puede entenderse sólo en términos del pasado o presente, sino también en términos de futuro, de las proyecciones, arquetipos, proyectos, mitos o metáforas con que la gente de una región o de un país se define e identifica frente a determinadas metas, horizontes de expectativas o utopías, por definición inalcanzables en su totalidad, aunque también por lo mismo fuertemente productivos y creados por medio de procesos de adaptación en el espacio y en el tiempo. (Zúñiga y Asún, 2003).

\section{Teoría de la acción comunicativa (Habermas)}

Si se tiene en cuenta que el concepto de inteligencia sintiente está estrechamente relacionado con las teorías sociológicas que afirman que los grupos humanos se estructuran con base en el ideal de un todo integral y, las formaciones sociales se fundamentan en la interacción de los mundos humanos, llámense éstos, objetivos, sociales o subjetivos, etc., tomando como referencia a Habermas (2001), se puede decir que toda persona tiene la capacidad de seguir e incidir en los procesos identitarios surgidos a lo largo de la historia del pensamiento que caracterizan al ser humano en tanto sujeto individual y social. Esta conformación de mundos se debe explicar como una posibilidad de realización humana, teniendo como eje articulador al lenguaje de la acción de las comunidades, de donde se deduce que bien puede relacionarse con la competencia comunicativa, al servir como instrumento de socialización entre los grupos humanos, situados en un espacio social y cultural y en un tiempo determinados, con necesidades de comunicación e interacción concretas en las que el lenguaje juega un papel central, pero cuyo uso resulta determinado por las características del contexto. (Pérez, 1999: 63).

Al ser el lenguaje el fundamento de la acción comunicativa, el habla y la escritura son la esencia del discurso y son éstos los que posibilitan la identificación de roles sociales en los diversos contextos en los que se desenvuelve que, según Habermas, se pueden sintetizar en dos: el Mundo de la Vida y el Mundo de los Sistemas, cuyas estrategias de acción social convergen en hacer explícitos los actos a través de la racionalidad argumentativa y la identificación de universos simbólicos en el proceso de la comunicación o interacción simbólica, teniendo como puente al lenguaje. Para representar más claramente esta idea, se propone en la Gráfica 1 la síntesis de lo anterior como estructura paradigmática:

Gráfica 1. Paradigma sociolingüístico (Teoría de la Acción Comunicativa-Habermas)

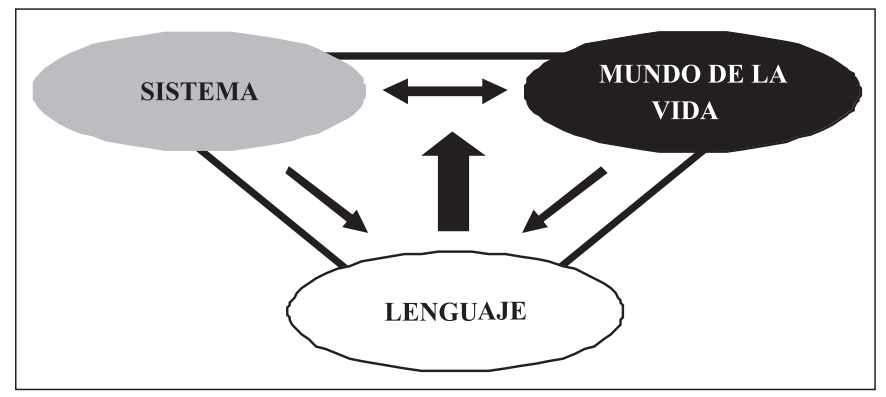


El Mundo de la Vida, categoría definida por Husserl (1992), quien la entiende como el horizonte último de sentido nunca agotable ni trascendible, en Habermas debe definirse como fundamento incuestionado de la concepción natural del mundo, porque en él vive cada persona su mundo subjetivo con las demás personas, creando mundos intersubjetivos vinculados por valores comunes y procesos de interpretación conjunta. Esto explica que en el Mundo de la $V i d a$, también cabe el mundo cultural, por ser el universo que da significación a los sujetos; es decir, es una red tejida por los sentidos que originan las acciones humanas y que los sujetos interpretan sobre sus ambientes y entornos con el fin de orientarse y conducirse en ellos.

Habermas (2001) plantea el concepto de Sistema como un fenómeno social trans-subjetivo, una situación de comunicación que se encuentra más allá de la subjetividad y es capaz de colonizar el Mundo de Vida. Plantea también, que la acción comunicativa dentro de los Sistemas debe verse como una meta de la sociedad, en la que el sujeto, por naturaleza autocrítico, puede abstraerse de la manipulación por medio de la racionalidad comunicativa, donde la interacción simbólica se dé partiendo de la buena fe de los hablantes, así como de la comprensión y entendimiento de las actitudes y símbolos de los mismos, buscando siempre llegar a acuerdos o consensos. Es decir, el camino sugerido por Habermas, busca analizar la sociedad desde dos formas de racionalidad: la sustantiva del Mundo de la Vida y la racionalidad formal del Sistema. Además, el filósofo alemán se propone demostrar que las personas necesitan de una Teoría de la Acción Comunicativa si su deseo es abordar de una forma adecuada, la problemática de la racionalización social. (Habermas, 2001: 24)

Se hace necesario subrayar que Habermas desarrolla su propuesta epistemológica en torno a las ciencias sociales y en comunión con la Escuela de Frankfurt. ${ }^{66}$ Entendiendo que la acción comunicativa es una metodología de la acción mediada por el lenguaje para establecer nexos entre el Mundo de la Vida y los Sistemas desde la racionalidad, se puede decir que se enfoca hacia el análisis de las estructuras generales de la acción orientada hacia el entendimiento como procedimiento, no como teoría del conocimiento, y además permite develar los cambios sociales a través de la explicación de los contextos.

Ahora bien, asumiendo que es la sociedad la que conforma tanto el Mundo de la Vida como los Sistemas, categorías ambas pobladas por miembros de grupos sociales, es posible definirla como un todo integral que contextualiza el entendimiento de los mundos objetivos, sociales e intersubjetivos. Habermas (2001) afirma al respecto: Todo acto del entendimiento que puede entenderse como la parte de un proceso cooperativo de interpretación que tiene como finalidad la obtención de definiciones de la situación que pueda ser intersubjetivamente reconocida. (Vol. 1: 104). Es así como la constitución del sujeto social sólo se da en la interacción entre los sujetos con la realidad vivida y se explica en la racionalidad argumentativa; esto es, en la capacidad para el lenguaje y la acción. Sólo de esta manera es posible comprender la forma

66 Es en la Escuela de Frankfurt en Alemania, donde se acopian investigaciones realizadas por diversos sociólogos, psicólogos, filósofos y economistas de la corriente neomarxista, con el objetivo de renovar la teoría marxista y subrayar la importancia de los estudios interdisciplinarios y la reflexión filosófica sobre la práctica científica. Se considera que Habermas es el último miembro de esta Escuela. 
como se construyen las sociedades a partir de acciones de individuos que no sólo comparten la misma normatividad sino también la intencionalidad de un universo simbólico común con el que se identifican a través del lenguaje, descubriendo el entramado del tejido social, condición esencial de la constitución y estructuración de las representaciones colectivas.

Es en este movimiento oscilante donde se revelan el Mundo de la Vida y el Sistema, como categorías que intervienen directamente en el comportamiento y asunción de roles. Es decir, la relación razón / cultura es una manera de tipificar a los sujetos que participan de la acción en el colectivo social, así como de orientar la intencionalidad de la acción. Para lograr esto, asegura Habermas (2001), se necesita del entendimiento, el cual debe apreciarse como un proceso de convencimiento recíproco que coordina las acciones de los distintos participantes sobre la base de una motivación razonada y conducente a acuerdos válidos contextualizados en la realidad misma.

La Teoría de la Acción Comunicativa en tanto análisis de la teoría sociológica de la acción, se expresa como aquella posibilidad que, según la afirmación de Habermas (2001):

Permite acceder a complejos temáticos: el concepto de racionalidad comunicativa que busca hacer frente a las reducciones cognitivo-instrumentales que se hacen de la razón, el concepto de sociedad articulado en dos niveles en los cuales asocia los paradigmas de Mundo de la Vida y Sistema. (Vol. 1 - 10)

Retomando el esquema del paradigma propuesto para este trabajo, lo anterior puede visualizarse en la Gráfica 2:

Gráfica 2. La Realidad, base epistémica de la Teoría de la Acción Comunicativa

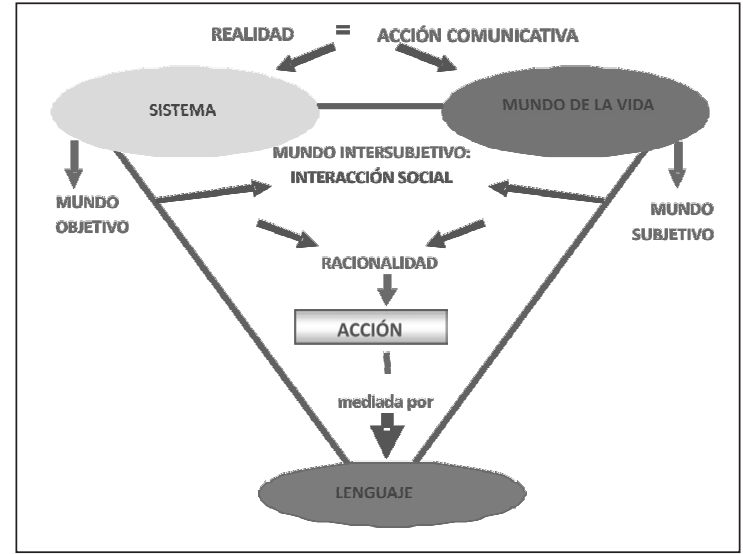

La Teoría de la Acción Comunicativa constituye entonces una nueva interpretación que fundamenta a las ciencias sociales y a su vez sirve como preámbulo de sus prácticas, las que en su afán estratégico por resolver problemas y transformar situaciones, proponen diferentes procedimientos que pasan a ser significativos en relación con la acción. 


\section{Narrativa}

Genéricamente, explica Barthes (1974), se puede definir la narrativa como la forma de relatar unos hechos ocurridos en un tiempo y en un lugar determinados, los que encadenados logran una nueva significación. Las actuales tecnologías están obligando a un cambio en las prácticas de la escritura social. La denominada Sociedad de la Información exige una nueva estructuración de los sistemas de comunicación y por ende, de presentación de los estudios de las culturas.

Con base en la definición antes expuesta, se puede decir que al igual que la narrativa tradicional, la narrativa digital busca contribuir a la comprensión, descripción, valoración y análisis de unos hechos, pero ahora acompañados de tecnologías digitales de la comunicación y, más específicamente, al uso del sistema de marcos. Desde saberes sociales como la antropología, la sociología, la comunicación, la literatura y en general el arte, se manejan discursos que antes era dispendioso procesar y organizar, pero que hoy, gracias a diversos programas de informática, así como a las redes de comunicación donde es posible procesar más fácilmente los datos cualitativos y la distribución de archivos, anteriormente sometidos a condiciones específicas de espacio y de tiempo, se facilitan dichos procesamientos y organizaciones.

La narrativa digital, según Rodríguez (s/f), es una forma de textualidad digital en la que los vínculos electrónicos unen fragmentos de textos, que pueden adoptar las formas del lenguaje a través de palabras, imágenes, sonidos, vídeos, etc., lo que de suyo conlleva una lectura diferente, a la que bien podría denominarse multilineal, multisecuencial o no lineal. Esto permite que lo que antes se consideraba como función exclusiva de un narrador, también pueda ser adoptada por un lector como coautor y copartícipe del texto narrativo, en cuanto tiene la posibilidad de hacer sus propias relaciones y de realizar sus propios enlaces, ampliando "a su manera" el campo contextual de la obra.

Dice Kendall (1995), que la narrativa digital se puede definir como un objeto virtual capaz de poner en dinámica no sólo una dimensión técnica (utilización de recursos audiovisuales y de nuevas tecnologías de la comunicación), sino también una dimensión estética; esto es, la posibilidad de afectar la inteligencia (en este caso sintiente) a partir del uso de tecnologías, haciendo posible entretejer un tipo especial de relato digital.

Las denominadas Redes de Comunicación tienen un papel definitivo, pues de manera explícita o implícita modifican los entornos, los hábitos y las prácticas sociales. Hoy son muchos los libros y documentos digitales que pueden ser consultados a través de la Internet y así lo demanda la tecnología de edición que maneja la Sociedad de la Información, permitiendo con ello muchas bondades, entre las que cabe destacar la reducción de costos en relación con los procesos tecnológicos que exigen los sistemas de edición tradicionales. (D'Sousa, s/f). 


\section{Narrativa histórica y cultural}

El objetivo de esta forma de narrar se dirige a la reconstrucción de los hechos históricos y culturales desde su fundación hasta la actualidad. Según Jitrik (1995), en este tipo de narraciones lo que se busca es reunir orgánicamente el pasado y atribuirle, en este marco, determinada racionalidad. $O$, dicho de modo más elemental, de aquello que ocurrió una parte es presentada de modo tal que se entiende o se debe entender por qué ocurrió. La historia y las manifestaciones culturales se constituyen aquí en el fundamento que nutre el campo de representación.

\section{Narrativa ambiental}

La pretensión de esta narrativa se dirige a abordar los contextos identitarios en los que un contexto funge como actor principal con inquietudes ambientales tales como las deficiencias higiénicas y sanitarias, falta de infraestructuras, problemáticas de viviendas y escuelas, destrucción o mala explotación de los recursos naturales, entre otros. En esta narrativa no se desconoce que en la actualidad, el concepto de ambiente es cada vez más amplio, al estar ligado a la jerarquía de valores de los individuos y las sociedades; según el Informe Brundtland (1987) el concepto de ambiente tiene una relación directa con la calidad de vida de las sociedades, con la limitación de los recursos naturales y con la degradación física del Planeta.

\section{Narrativa administrativa y económica}

El objetivo que se desarrolla en esta narrativa es la búsqueda de la identidad regional, apoyada en elementos misceláneos como, por ejemplo, ideas sobre la explotación de la naturaleza y el paisaje, el medioambiente construido, la cultura, la etnicidad y la pertenencia al territorio, el éxito económico, la pobreza, las fronteras entre el nosotros y el ellos, las historias y utopías contextualizadas en prácticas, rituales y discursos que faciliten la expresión del manejo económico y administrativo de las comunidades (Anderson, 2000). Esta narrativa, en palabras de Amtmann (1997:9), tiene un compromiso afectivo vital con el pasado, presente y futuro de los procesos económicos-sociales y culturales que acaecen en una localidad o región.

\section{Narrativa Turística}

El objetivo de esta narrativa es mostrar que el turismo es una experiencia co-creativa que representa un motor, además de económico, emocional (para este trabajo, sintiente), según Pine y Gilmore (1999). La hipótesis de MacCannell (1988) es que lo que motiva al turista a desplazarse es el deseo de experimentar interacciones auténticas con otros, durante las cuales se tenga acceso a apreciar la vida cotidiana en un entorno diferente. Según el mismo autor, una de las principales tendencias del turismo es fomentar la restauración, la conservación y la recreación ficticia de los grupos sociales. Para MacCannell, estas formas se dan en tanto todos los grupos del mundo están situados en una red de interacciones global; por lo tanto, esta narrativa se enfrenta a una expresión simbólica orientada hacia un fin y un valor de uso dentro del sistema global. 


\section{Inteligencia artificial y sistema de marcos}

Explica Maldonado (1998) que desde la filosofía, la Inteligencia Artificial se hizo posible cuando se superó la concepción dual mente-cuerpo, proceso de largo aliento sustentado en tres teorías básicas: 1 . El pensamiento se manifiesta como actividad física y como tal puede ser desarrollado tanto por el cerebro como por máquinas que lo emulen. 2. Los procesos de conocimiento son caracterizables desde las matemáticas. 3. El cerebro se desempeña como un sistema que procesa información y, visto así, el computador es su metáfora. Citando a Frankin, Maldonado ubica los estudios de inteligencia artificial, afirmando que lo hace dentro de las dimensiones de lo posible, antes de caracterizar el cómo de los estudios que caben dentro de los difusos límites de este campo, a saber:

- La inteligencia es un continuo que se muestra en los seres en grados, contrario a la forma del todo o nada.

- La inteligencia de un agente depende de la integración de componentes y se realiza, en la práctica, de manera parcial.

- La activación de la inteligencia se lleva a cabo por conjuntos de mecanismos que se ubican en un entorno.

- El fluido continuo de la acción absorbe totalmente la inteligencia: el sistema inteligente está permanentemente determinando qué hacer en seguida.

- La inteligencia opera sobre las sensaciones para generar información para su propio uso.

- La inteligencia usa información almacenada en memoria para generar acciones mediante un proceso reconstructivo, más que por simple recuerdo.

- La inteligencia se puede representar de manera limitada e incremental: las máquinas del futuro serán más inteligentes que las actuales.

Gracias a la inteligencia artificial, término acuñado en 1956, es posible construir procesos cognitivos, representativos, perceptivos o de acciones que al ser ejecutados sobre una estructura física, son concientes de sí mismos y producen resultados conducentes a rendimientos secuenciales de entradas que además pueden ser almacenados en dicha estructura. Explican Haton y Haton (1991) que en la actualidad ese saber multidisciplinario que se ocupa de investigar muestra dos tendencias básicas: 1 . Centrada en la simulación de los mecanismos propios de la inteligencia. 2. Tiende a emular las capacidades instintivas de la inteligencia, como la percepción, la conceptualización, el razonamiento y la toma de decisiones. Obviamente, ambas son tributarias de una concepción teórica de la inteligencia humana.

Es interesante tener en cuenta que, para que la inteligencia artificial cumpla con su propósito fundamental, que según Tanimoto (1987) consiste en optimizar la comprensión que implica los procesos de razonamiento, aprendizaje y percepción humanas, no se debe disolver la tríada compuesta por la teoría, la metodología y el dispositivo, pues es la que permite representar el conocimiento, dirigir los procesos de búsqueda encaminados a la solución de problemas, a la percepción y a su inferencia. Aquí surge el concepto de agente, foco de la inteligencia artificial como muestra integral de dicha tríada, a la que incorpora el dinamismo de la acción y la interacción con el medio. Es el agente el que promulga por el uso de metáforas encaminadas a la solución de problemas. 
Russell y Norvig (1996) definen un agente como el objeto capaz de percibir un ambiente a través de censores respondiendo racional y autónomamente a través de efectores.

Cuando se colecta la información durante el proceso de adquisición de conocimiento, se requiere además, de una simulación, la cual se ve expuesta a partir de técnicas de programación, tales como los micromundos, las redes neuronales, el reconocimiento de patrones, el procesamiento de lenguaje natural, la interfaz hombre-máquina, las estructuras de datos, las bases de conocimiento, las redes semánticas, los grafos conceptuales, entre otros. Sólo entonces es posible representar el espacio, concepto fundamental cuando se requiere determinar un proceso de representación.

Con el surgimiento del movimiento de Inteligencia Artificial, Minsky (1975) desarrolla el concepto de sistemas de marcos para hacer representaciones de sistemas estructurados. La teoría de los marcos la explica el mismo Minski: Cuando nos enfrentamos a una nueva situación, o cuando realizamos un cambio sustancial en nuestra visión de un problema, seleccionamos en nuestra memoria una estructura denominada marco. Este es un esquema que nosotros recordamos y que debemos adaptar a la realidad cambiando los detalles según sea necesario. Es decir, si los seres humanos logran condensar y convencionalizar situaciones y complejidades del lenguaje y el pensamiento en palabras y símbolos compactos, los que organizados pueden enmarcarse (colocar dentro de un marco como espacio sugerido) para representar situaciones estereotipadas y relacionarlas estructuralmente a manera de sistema que representa acciones, entonces se encuentran diversas formas relacionales que dan lugar al hipertexto.

\section{PROCESO METODOLÓGICO}

La presente aproximación documental se realiza con el fin de dar cuenta de las temáticas que son motivo de estudio para el diseño, construcción, elaboración y desarrollo de la Línea de Investigación del Doctorado en Gestión de Conocimiento, Inteligencia Sintiente e Identidad Cultural, partiendo de los referentes teóricos, las delimitaciones estructurales y contextuales, además del reconocimiento de aquellos autores que las han asumido.

Para la elaboración de este artículo se tuvo en cuenta la producción intelectual en la cual se consideran factores tales como su carácter empírico, los procedimientos metodológicos empleados para su realización, los enfoques teóricos fundamentales, las estrategias metodológicas que se emplean, las preguntas que lo direccionan y los resultados que se espera obtener. Para ello se consideran los documentos de soporte antes enunciados por concebir que sirven como apoyo para la construcción no sólo del presente artículo sino también de los proyectos que se sumarán a la línea de investigación doctoral.

El rastreo de la documentación se realizó teniendo en cuenta los siguientes descriptores temáticos básicos: El concepto de meta-sistema y el sistema de marcos, inteligencia sintiente, identidad cultural, Teoría de la Acción Comunicativa y el concepto de narrativas y tipos de narración que darán inicio y continuidad al proyecto. Para la lectura, categorización y resumen 
de la documentación seleccionada, se previó que en la medida en que se hizo la lectura de cada documento, se fue resumiendo y clasificando según las temáticas donde se iba ubicando la información, extrayendo de ellas las ideas centrales que lo sustentan. De esta forma el proyecto se orienta a crear condiciones discursivas, gráficas, auditivas, etc., conducentes a sugerir relaciones interculturales desde una meridiana percepción del pasado, que explique las experiencias presentes y desde el cual pueda vislumbrarse un futuro competente.

Ahora bien, considerando que la pretensión de la Línea de Investigación del Doctorado en Gestión de Conocimiento a la que se dirige este documento a través de esta aproximación documental, es gestionar conocimiento a partir del existente para generar nuevo conocimiento y siendo evidente que lo aquí expuesto no es un producto terminado, sino un punto de partida para la realización de constantes búsquedas, también se concibe como una dinámica propia del ejercicio mismo de investigar, así como el enfoque y la manera de entender sus temáticas. Por consiguiente, es posible encontrar nuevos estudios que aporten a lo expuesto en el presente artículo.

\section{RESULTADOS ESPERADOS}

Esta aproximación documental, entendida como una selección del universo de la documentación existente, surge con el fin de contribuir en el desarrollo de la línea Doctoral para su direccionamiento desde la Línea de Investigación Inteligencia Sintiente e Identidad Cultural. A partir de dicha aproximación se espera:

1. Servir como punto de partida, para gestionar conocimiento comprensivo sobre la recolección de la información, representada a manera de hiperlibro.

2. Aunar las bases conceptuales que aseguren la continuidad de los proyectos que se van a llevar a cabo desde la línea de investigación y su comprensión sintética para las personas involucradas.

3. Asociar los mecanismos de la memoria con los eventos hipertextuales, donde pasado, presente y futuro se actualicen narrativamente en tiempos y espacios globalizantes que permitan la actuación desde lo local.

4. Generar documentos que se apoyen tanto teórica como temática y metodológicamente para el logro de la competencia investigativa conducente al planteamiento de sus futuras tesis de grado para los estudiantes del Doctorado en Gestión de Conocimiento.

\section{CONCLUSIONES}

La estructura del hipertexto potencia la intertextualidad a límites insospechados. Puede llegar a sustituir la tradicional triada de los estudios basados en narrativas: autor, obra, tradición, en favor de la triada: texto, discurso, cultura, liberando así el texto de determinantes psicológicos, sociológicos o históricos y abriéndolo a infinitas relaciones. 
Por estar el hipertexto constituido por cuerpos de textos conectados sin eje organizador, el sistema puede centrase según el interés personal y el lector tendrá la posibilidad de seleccionar el centro de interés sin encerrarse en un sistema de organización o jerarquía.

El formato del hipertexto, aunque sigue de alguna manera la línea de otras tecnologías contemporáneas, (radio, televisión, o video), transforma radicalmente tanto los sistemas de acceso y almacenamiento de información, las prácticas de lectura y escritura, como los modos y medios de interacción comunicativa, constituyéndose en un nuevo dispositivo de lenguaje.

La hipertextualidad puede verse como una nueva narrativa hecha de fragmentos, de voces venidas de otros textos, de otros códigos, de significados siempre diferidos en redes infinitas de textos, surgiendo una nueva narratividad cuyo goce estético se encuentra inmerso en el mismo fragmento. De igual manera, la potencialidad explicativa del sistema de marcos se basa en la inclusión de expectativas.

El hiperlibro enmarcado en forma de narrativas, consolida un espacio (en este caso institucional) y facilita la comunicación e interacción para transferir y extender propuestas, investigaciones y resultados tanto para la institución como para las comunidades académicas. La propuesta apunta a una nueva forma de narrar desde la misma realidad vista con una inteligencia sintiente, plasmando en él las memorias y las experiencias humanas.

A partir de la construcción del referente teórico referenciado y de los resultados de investigaciones evidenciados, se alimenta la línea de investigación doctoral, genera nuevas preguntas de investigación y se hace posible adelantar estudios pertinentes y de calidad, destinados a avanzar en la construcción teórica en el campo de la gestión de conocimiento desde las realidades ya analizadas por otros investigadores.

\section{REFERENCIAS BIBLIOGRÁFICAS}

AMARAL, Kimberly. Hypertext and writing: an overview of the hypertext medium. En: http://www.umassd.edu/Public/People/kAmaral/Thesis/hypertext.html.

AMTMANN, C. (1997) "Identidad regional y articulación de los actores sociales en procesos de desarrollo regional”. En: Revista Austral de Ciencias Sociales No.1, pp. 5-14.

ANDERSON, B. (2000) Comunidades imaginadas: reflexiones sobre el origen y la difusión del nacionalismo. Ciudad de México: Fondo de Cultura Económica.

BARAN, Paul (1964). On Distributed Communications. MEMORANDUM RM-3420-PR. http://www.rand.org/publications/RM/baran.list.html.

BARTHES, R. y otros (1974), Análisis estructural del relato. Buenos Aires, Tiempo Contemporáneo, 1974. 
BARTHES, Roland. (1980) S/Z. Madrid: Siglo XXI.

BORGES, Jorge Luís. (1975). "La biblioteca de Babel”. En: Ficciones. Barcelona: Círculo de Lectores.

BRUNDTLAND, G. A. (1987) Nuestro futuro común. Madrid: Alianza.

BUSH, Vannevar. (1945). Cómo podríamos pensar. En: http://biblioweb.sindominio.net/ pensamiento/vbush-es.html.

BUSH, Vannevar: "As We May Think" in The Atlantic Montly, july 1945. En: http://www. theatlantic.com/doc/194507/bush.

CASTELLS, M. (1999) 'Globalización, Identidad y Estado en América Latina', Revista del PNUD.

DERRIDA Jacques (1972). Dos ensayos. La estructura, el signo y el juego en el discurso de las ciencias humanas. El teatro de la crueldad y la clausura de la representación. Barcelona: Cuadernos Anagrama.

D'SOUSA, Carmen. Redes de Comunicación. En: http://www.monografias.com/trabajos11/ reco/reco.shtml\#bi.

GARCÍA CANCLINI, Néstor. Consumidores y ciudadanos. Grijalbo, México, 1995.

HATON, J-P y HATN, M-C. (1991) La inteligencia artificial. Una aproximación. Buenos Aires: Paidós.

HABERMAS, Jürgen. (2001). Teoría de la acción comunicativa. 2 vols. Madrid: Taurus. $2^{\mathrm{a}}$ ed.

HERRERA, Gloria. (2008). Las universidades en las organizaciones inteligentes en la Sociedad del Conocimiento. Presentación. En: http:/www.slideshare.net/oasconval/ unad-congreso-gloria-herrera.

HOYOS BOTERO, Consuelo (2000). Un modelo para investigación documental. Medellín: Señal Editora.

HUSSERL, Edmund. (1992). Invitación a la fenomenología. Barcelona: Paidós.

JITRIK, Noé. (1995) Historia e imaginación literaria. Las posibilidades de un género. Buenos Aires: Biblos. 
KENDALL, Robert. (1995). Writing for the new millennium. The birth of electronic literature. En: Poets \& Writers Magazine (Nov./Dec.). En: www.wordcircuits.com/kendall/essays/ pw1.htm.

KERNAN, Alvin. (1996) La muerte de la Literatura. Caracas: Monte Ávila.

KRISTULA, Dave (2001). The History of Internet. En: http:/www.davesite.com/webstation/ net-history.shtml.

LANDOW, George P. (1995) Hipertexto. La convergencia de la teoría crítica contemporánea y la tecnología. Barcelona: Paidós.

LANDOW, George P. (1997) Teoría del hipertexto. Barcelona: Paidós.

LEINER, Barry M. y otros. (2005). A brief history of the Internet. En: http://www.isoc.org/ internet/history/brief.shtml.

LOS ESPACIOS HIPERTEXTUALES: la arquitectura de la información. En: http://www. dialogica.com.ar/clicsmodernos/2003/09/8-los-espacios-hipertextuales.html.

MACCANNELL, Dean (1988) "Turismo e identidad cultural". En: Todorov, T. y otros, Cruce de culturas y mestizaje cultural. Barcelona: Júcar.

MALDONADO, Luís Facundo (1998). Línea de inteligencia artificial y procesos de razonamiento. Universidad Pedagógica Nacional. TED: Tecné, Episteme y Didaxis. Bogotá, pp. 19-40. En: http://www.pedagogica.edu.co/storage/ted/articulos/ted03_04arti.pdf.

MALDONADO, Luís Facundo. LANDAZABAL, Diana. HERNANDEZ, Juan. GUZMAN, Maria. ROJAS, Patricia. MUÑOZ, Isabel. GONZALEZ, Almeida (2008) Gestión de conocimiento, visibilidad del desarrollo científico. ISBN 978-958-651-483-5.

MINISTERIO DE CULTURA. Plan Nacional de Cultura 2001-2010: Hacia una ciudadanía democrática cultural un plan colectivo desde y para un país plural. En: http://www. mincultura.gov.co/VBeContent/newsdetail.asp?id=371\&idcompany=3.

MINSKY, Marvin (1975). A framework for Representing Knowledge, Winston. P. Ed. The Psychology of Computer Vision. Nueva York: MacGraw-Hill.

NELSON, Theodore. (1967). Literary Machines. (Paperback).

NELSON, Theodor Holm. Ted Nelson Homepage. En:http://ted.hyperland.com/ ONG, Walter. (1987) Oralidad y escritura. Tecnologías de la palabra. México: Fondo de Cultura Económica. 
PÉREZ ABRIL, Mauricio. (1999) “Competencia textual, competencia pragmática y competencia argumentativa". En: BOGOYA Daniel. Hacia una cultura de la evaluación para el siglo XXI. Bogotá: Universidad Nacional de Colombia.

PINE, B.J. y GILMORE, J.H. (1999). The experience economy. Work in theatre and every business a stage. Boston; Harvard Business Press.

PORUNPUÑADODEHIPERLIBROS.En:http://www.dialogica.com.ar/clicsmodernos/2003/09/6por-un-punado-de-hiperlibros.html.

PROJECT XANADU. The Original Hypertext Project. http://www.xanadu.net.

RODRIGUEZ, José Alejandro. El relato Digital. Pontificia Universidad Javeriana. Bogotá. En: La narrativa Digital. http://www.javeriana.edu.co/Facultades/C_Sociales/Facultad/ sociales_virtual/publicaciones/relatodigital/accesos/relato_digital.html.

ROJAS, Alejandro. (1998) La inteligencia artificial y la filosofía. En: Revista Análisis. Bogotá: Universidad Santo Tomás, enero-julio.

RUSSELL, S. y NORVIG, P. (1996). Inteligencia Artificial Un en foque moderno. México: Prentice Hall.

TANIMOTO, S. L. (1987). The elements of Artificial lntelligence: An introduction using LISP. Rockville, Maryland: Computer Science Press.

XANADU AUSTRALIA. Xanadu: The Information Future. http://ww.aus.xanadu.com/ xanadu/future.html.

ZUBIRI, Xavier. Inteligencia sentiente. Inteligencia y realidad. Madrid: Alianza Editorial Sociedad de Estudios y Publicaciones. 1980.

ZUBIRI, Xavier. (1984) "El problema teologal del hombre". En El hombre y Dios. Madrid. Alianza, pp. 369-383.

ZUBIRI, Xavier. (2005) ¿Qué es investigar? En: www.zubiri.org/general/xzreview/2005/ pdf/zubiri_2005.pdf.

ZÚÑIGA, C. y R. ASÚN (2003). "Identidad regional en un contexto de cambio. Un estudio en la Araucanía, Chile”. En: Psicología Política No. 26, pp.73-92. 